\title{
Il team dell'accesso vascolare: istruzioni per l'uso
}

\author{
Franco Galli ${ }^{1}$, Luigi Tazza ${ }^{2}$, Luciano Carbonari ${ }^{3}$
}

${ }^{1}$ Già Responsabile dell'attività di Chirurgia dell'Accesso Vascolare per Emodialisi presso la Divisione di Nefrologia, Fondazione S. Maugeri, Pavia

${ }^{2}$ UO Dialisi, Dipartimento di Scienze Chirurgiche, Policlinico A. Gemelli, Roma

${ }^{3}$ SOD Chirurgia Vascolare, Azienda Ospedali Riuniti di Ancona, Centro di Riferimento Regione Marche per gli Accessi Vascolari in Emodialisi

\begin{abstract}
VASCULAR ACCESS TEAM: OPERATING INSTRUCTIONS
Abstract. The management of vascular access by the nephrologist is increasingly difficult. A great help could come from the vascular access team, which is composed of various specialists and can face the complex and different issues of hemodialysis access under the coordination of the nephrologist. The team is a tool that every nephrologist that deals in his facility with vascular access management should have. The vascular access team should be built by identifying useful competences. The inability to form a local team, should never prevent the nephrologist to refer to another, external team able to solve vascular access problems.
\end{abstract}

Key words: Hemodialysis, Vascular access, Vascular surgery, Interventional radiology

Conflict of interest: None.

Ricevuto: 21 Gennaio 2013; Accettato: 22 Febbraio 2013

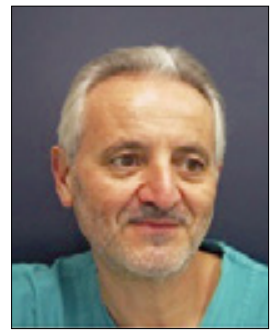

Franco Galli

\section{Riflettere sorridendo}

Si racconta che un vecchio boscaiolo canadese, convinto dalla pubblicità radiofonica, decide di acquistare per la prima volta in vita sua una motosega. Non appena ne entra in possesso, raggiunge il bosco per verificare se quell'attrezzo, per lui nuovo, sia migliore della tradizionale sega a mano usata fino al giorno prima. La sera, però, è stanchissimo e, soprattutto, deluso, perché la quantità di legna tagliata è di gran lunga inferiore rispetto al solito. Così, l'indomani si reca dal venditore per esporre le sue rimostranze; viene convocato un tecnico che, impugnata la motosega, la mette subito in moto per una verifica. Il rombo del motore fa sussultare il boscaiolo che chiede d'istinto: "Ma che cos'è questo rumore?".

Nella gestione dell'accesso vascolare il rischio di non utilizzare gli strumenti adatti o, peggio, di usarli in maniera non appropriata è forse più grande di quanto si pensi. Riflettiamoci.

\section{Premessa}

Gestire l'accesso vascolare significa farsi carico di tutte le problematiche che tale irrinunciabile strumento del trattamento emodialitico può presentare, dalla progettazione alla realizzazione e al mantenimento. La cura di un paziente in dialisi, in generale, richiede di affrontare quotidianamente un susse- guirsi di situazioni che non troverebbero soluzione senza la disponibilità di determinati strumenti; non è sufficiente, però, disporne, ma bisogna anche conoscerli bene, perché, fra i tanti e difficili compiti del gestore, vi è anche quello di operare scelte a nome e per conto di chi non è in grado di farlo. In dialisi, in particolare, ciascun paziente cronico demanda di fatto al suo nefrologo l'individuazione e la scelta dei percorsi più utili per assicurargli non solo la sopravvivenza ma anche una vita qualitativamente accettabile e, dal momento che i trattamenti sostitutivi dell'IRC sono tanto più efficaci quanto più sono personalizzati, è necessario prendere decisioni sempre più "su misura". Fra le numerose scelte imposte dalla dialisi vi è quella che riguarda il tipo di accesso vascolare; questo, infatti, deve rappresentare la migliore soluzione per quel paziente in quel particolare momento del suo percorso di malattia e del suo trattamento. Il paziente, dal canto suo, non è quasi mai in grado di scegliersi la strategia terapeutica migliore e, soprattutto per quanto concerne l'accesso vascolare, senza specifiche conoscenze potrebbe essere indotto a optare per soluzioni forse più comode ma spesso non coincidenti con quelle per lui più utili. Al nefrologo, allora, spetta la prerogativa di giudicare e di operare, per delega del paziente, nel modo migliore, avendo però sempre presente che gli strumenti "giusti" nelle mani "sbagliate" possono sortire l'effetto contrario.

Non è certamente facile giudicare dall'esterno, ammesso che sia importante farlo, se un centro dialisi svolga una politica dell'accesso che possa considerarsi accettabile; mancano in realtà indicatori affidabili e obiettivi e quelli spesso adottati non esprimono la reale valenza della politica di cui sopra. Se 
decidessimo, per esempio, che la percentuale media dei CVC nei pazienti prevalenti italiani, segnalata, per esempio, dall'ultimo studio DOPPS, è lo spartiacque fra centri "virtuosi" e centri "viziosi", forse rischieremmo di sottostimare sia gli uni sia gli altri. Il centro che, a fronte di una percentuale di CVC anche leggermente superiore rispetto a quella indicata dallo studio, registra un rateo di complicanze trombotiche e infettive documentate molto basso rientra forse tra $\mathrm{i}$ virtuosi, quasi a dire: avranno anche tanti CVC ma li sanno gestire molto bene. Per contro, il centro che si ostina a tenere molto alto il numero dei CVC cronici, pur avendo ottime opportunità di incrementare la confezione delle FAV, non ha certo un comportamento virtuoso. La percentuale di CVC nei pazienti prevalenti potrebbe essere uno dei possibili indicatori di politica dell'accesso vascolare ma non certamente l'unico e il più attendibile e, soprattutto, non esprimerebbe le reali condizioni organizzative (o disorganizzative) di un centro.

Ben venga allora l'indagine su scala nazionale, messa a punto da D. Bonucchi, che il Gruppo di Studio degli Accessi Vascolari sta licenziando e che dovrebbe dare una fotografia in tema di accessi sufficientemente nitida della nostra realtà; solo con quella potremo finalmente confrontarci con il resto del mondo e capire se la fama di buoni gestori che ci ha attribuito finora lo studio Dopps è ancora meritata.

\section{Requisiti necessari per una corretta gestione dell'accesso vascolare}

Qualsiasi tipo di gestione è impraticabile se manca lo strumento principe per garantirne il funzionamento; tale strumento si chiama "cultura".

La conoscenza della materia "accesso vascolare" dovrebbe iniziare, prioritariamente, durante la formazione specialistica di base (scuola di specialità) che include, nel suo programma, tale insegnamento. Abbiamo usato il condizionale solo per significare che non tutte le scuole riescono purtroppo a fornire tale insegnamento perché spesso sono prive di tradizione e di competenza nefrologica in materia; qualcosa di analogo avviene anche nelle altre scuole di specialità non nefrologiche (Chirurgia Vascolare, Radiologia), che dovrebbero comunque trattare tale l'argomento visto il sempre maggiore coinvolgimento di tali specialità nella tematica dell'accesso.

Il Gruppo di Studio degli Accessi Vascolari della SIN ha, di recente, avviato un progetto di formazione che prevede, tra l'altro, di rilanciare attraverso la Società Italiana di Nefrologia l'invito accorato ma deciso a tutte le scuole di specialità perché l'insegnamento sulle tematiche inerenti l'accesso vascolare per emodialisi sia assicurato in forma adeguata e completa a tutti gli specializzandi. Solo con una preparazione di base corretta e completa, le attitudini e le vocazioni dei giovani colleghi possono essere incanalate nei percorsi formativi successivi che vanno, a seconda delle esigenze, dai corsi di aggiornamento/perfezionamento a veri e propri master universitari. Lo stesso invito, seppur con un taglio diverso, andrebbe rivolto anche alle altre scuole di specialità e ai corsi di laurea in scienze infermieristiche. Investire sulla formazione in tema di accesso vascolare soprattutto in ambito nefrologico è necessario, in quanto vi è un patrimonio di conoscenze ed espe- rienze su tale argomento, prodotto nel tempo da nefrologi e cultori della materia oggi non più in attività o nell'imminenza della pensione, che rischia di andare disperso se non trasmesso alle nuove generazioni; queste, senza i necessari strumenti, saranno costrette ad affidare ad altre figure professionali la risoluzione (o la non risoluzione) dei problemi generati dall'accesso vascolare. Vi è anche una legittima ragione di orgoglio nel progetto formativo: cercare di mantenere viva un'attività peculiare della nefrologia tramandandola.

Quanto finora asserito concerne un presente rivolto al suo possibile (auspicabile) futuro ma vi è anche un presente che guarda al presente e che cerca di indicare, a chi è già in cammino, gli strumenti senza i quali è impossibile gestire correttamente gli accessi di oggi; gli arnesi a cui ci si riferisce non sono tanto i moderni dispositivi o le apparecchiature sofisticate quanto le collaborazioni con specialisti di altre discipline.

\section{La borsa degli attrezzi: "il team degli accessi vascolari"}

È da tempo che il Gruppo di Studio enfatizza la necessità per il nefrologo, dedito ai pazienti dializzati, di mettere insieme nel proprio ambito lavorativo il team degli accessi vascolari divenendone possibilmente il motore. Il team, in realtà, non è uno strumento unico ma è una sorta di kit che contiene strumenti diversi ciascuno dei quali svolge funzioni diverse; sta all'utilizzatore del kit, che ben conosce il problema, fare in modo di mettere in campo ciò che ritiene più utile in una determinata circostanza o, addirittura, di rendere uno strumento complementare a un altro. Quando, in un precedente editoriale, parlammo di modelli organizzativi giungendo all'identificazione del team degli accessi vascolari, di fatto proponemmo un modello di kit generico che avrebbe voluto ispirare l'assemblaggio di altrettanti kit, contenenti ciò che ciascun centro fosse in grado di inserirvi; ora è tempo di dare qualche ulteriore suggerimento, ispirato come sempre dall'esperienza personale, sul modo forse più utile, senza pretendere che sia il migliore, di utilizzare quel contenuto.

\section{Il kit di base}

\section{Lo strumento "infermiere"}

Il team dell'accesso vascolare è, di fatto, l'ampliamento di un mini-team che si identifica in quel gruppo di lavoro che, per motivi naturali e istituzionali, si crea all'interno di ciascun centro dialisi; esso è composto dal personale infermieristico e dal nefrologo che lo guida. Esso deve, anzi dovrebbe, usiamo pure il condizionale, essere la massima espressione di lavoro collaborativo fra due componenti che hanno compiti diversi. Non sono, ovviamente, ruoli intercambiabili, soprattutto per quanto concerne le responsabilità, ma non è provocatorio affermare che, se l'infermiere non può fare il medico, quest'ultimo può fare l'infermiere, se occorre, e deve saperlo fare bene. Solo accogliendo tale principio, è assolutamente raccomandabile che, in tema d'accesso vascolare, tutto ciò che "fa" l'infermiere (e quasi sempre lo fa molto bene) lo debba imparare prioritariamente dal medico esperto e non da altri infermieri; 
in questo modo, la continuità di formazione omogenea della componente infermieristica di un centro è assicurata. Il medico deve, pertanto, insegnare a utilizzare correttamente l'accesso; ci riferiamo in particolare all'infissione e, soprattutto, alla rimozione, con compressione, degli aghi da fistola, sorvegliando affinché le ragioni "logistiche" non prevalgano su quelle "cliniche".

Il team base dell'accesso vascolare deve essere un esempio di efficienza e funzionalità; per tale motivo è necessario che entrambe le componenti si mettano spesso in discussione identificando le criticità e/o la mancata adesione ai protocolli di gestione interni; è scontato che, nel team, il nefrologo debba sempre motivare il personale infermieristico ponendosi come esempio da seguire e non da evitare e lo può fare solo se la sua competenza in tema di accessi è assolutamente fuori discussione.

\section{Lo strumento "chirurgo vascolare"}

Chi meglio di un esperto chirurgo vascolare è più abile nell'isolare, nel dominare, nell'anastomizzare o nel liberare dai trombi i vasi? La risposta è scontata e non potrebbe essere diversamente se ci si riferisce a chi, professionalmente, ha familiarità con l'argomento. La storia ci ha fatto incontrare in un passato non lontano chirurghi vascolari illuminati e ispirati che da subito hanno saputo cogliere le esigenze e le difficoltà di nefrologi e pazienti e si sono dedicati con passione alla risoluzione di aspetti complessi dell'accesso vascolare; tali "personaggi" hanno costituito l'eccezione in un contesto di specialisti che fino a non molto tempo fa si sono mostrati generalmente poco interessati o, talvolta, ritrosi nei confronti delle istanze dei nefrologi. Oggi, il quadro è fortunatamente cambiato grazie a un maggior numero di specialisti chirurgi vascolari che hanno compreso che l'accesso vascolare non è l'intervento riempitivo di una lista operatoria ma qualcosa di più impegnativo. Chi lo fa con spirito collaborativo (e non attratto dal fattore DRG) ha, in genere, un ottimo rapporto con il nefrologo che, con altrettanto spirito collaborativo, cerca di mettere a frutto la superiorità tecnica del collega puntando su obiettivi difficili ma certamente utili per il paziente che ha in carico. Di esempi collaborativi funzionanti se ne conoscono diversi oggi in Italia ed è evidente che da tale tipo di joint venture non possa che derivare un vantaggio per i pazienti. Va anche però segnalato, per dovere di cronaca, che esistono situazioni di convivenza nefrologo-chirurgo vascolare imposte; come in tutte le convivenze forzate, mancando la sintonia manca la "libertà di collaborare", perché il nefrologo è costretto a inviare il paziente al chirurgo vascolare che, a sua volta, è costretto ad accoglierlo per farsene carico. Le collaborazioni "forzate" hanno purtroppo quasi sempre tiranni (= chi le impone) e vittime (= pazienti). Come usare bene allora il chirurgo vascolare? Condividendo il problema, stabilendo con lui i passi da compiere e da ultimo, ma non meno importante, dandogli il riscontro nel tempo del lavoro eseguito. Superata la vexata quaestio che ha caratterizzato i primi ufficiali rapporti fra Gruppo di Studio (dei nefrologi) e chirurghi vascolari relativa a chi dovesse confezionare l'accesso vascolare, oggi è salomonicamente accettato che "confeziona l'accesso chi lo sa fare", e proprio perché il nefrologo non è in grado di allestire ogni tipo di accesso, è obbligato, nei casi difficili, a ricorrere a competenze e ad abilità superiori alle sue. Non è possibile stabilire, sulla carta, i confini oltre i quali non deve avventurarsi il nefrologo; solo da una corretta collaborazione con il suo chirurgo vascolare di riferimento possono derivare le regole di comportamento. Un esempio: è inutile far perdere tempo al chirurgo vascolare per una fistola manifestamente facile, mentre è assolutamente necessario programmare il suo coinvolgimento prima che l'accesso diventi difficile. Coinvolgerlo in corso d'opera perché ci si trova in "braghe di tela" non è più collaborare ma "tirare bidoni", come si dice in gergo.

\section{Lo strumento "radiologo interventista"}

La letteratura scientifica si arricchisce sempre più di segnalazioni relative a procedure angioradiologiche effettuate per riparare accessi vascolari periferici malfunzionanti o per recuperare distretti venosi centrali un tempo dati per perduti. L'angioradiologia interventistica è, insieme all'ecografia, la novità più importante, nel campo degli accessi, di questi ultimi quindici anni. Tuttavia, mentre l'ecografo, presente ormai in tutti i centri nefrodialitici italiani, viene correntemente e correttamente usato dai nefrologi per tutte le necessità diagnostico-interventiste che l'apparecchio consente, il ricorso all'angiografo, attraverso lo specialista autorizzato, non è ancora diffuso come invece sarebbe auspicabile. La relazione fra danni venosi centrali e utilizzo di CVC è nota da tempo e finché l'interventistica radiologica non ha offerto la possibilità di recuperare distretti vascolari giudicati fino allora perduti, l'unica possibilità di costruire fistole laddove vi era un grave ostacolo al deflusso venoso era quella di ricorrere ai cosiddetti "accessi disperati”, creando percorsi extra-anatomici. L'interventistica radiologica, attraverso la riapertura di vasi altrimenti non utilizzabili, ha di fatto mandato in pensione, per fortuna dei pazienti, gli accessi disperati. L'angioradiologo è uno specialista la cui attività è in continua evoluzione perché in continua evoluzione sono i dispositivi che utilizza nelle sue procedure; non cambiano i principi su cui si fonda il suo lavoro, ma cambiano, invece, migliorando sempre di più, gli strumenti che usa. Ne deriva che l'angioradiologo sia attualmente uno degli strumenti più potenti a disposizione del nefrologo nella gestione degli accessi a patto però, anche in questo caso, che si stabiliscano con lui rapporti di collaborazione vera, propositiva. Per comprendere le potenzialità di questa branca specialistica c'è un unico modo: frequentare la sala angiografica (da semplici spettatori) e interagire con l'operatore sapendolo sapientemente convincere a mettere nel suo mirino il bersaglio che si vorrebbe raggiungere. Per farlo bisogna conoscere, attraverso la letteratura scientifica, quel tanto che serve a formulare proposte/richieste che possano essere accolte e convertite in utili procedure. Se ci si limita a inviare il paziente in sala angiografica senza pianificare con i colleghi radiologi la possibile procedura, non bisogna lamentarsi se viene eseguita una semplice procedura diagnostica o se l'interventistica utilizzata non porta ai risultati previsti. Fra gli angioradiologi che hanno affrontato problemi di accessi vascolari si sono creati vari "movimenti di pensiero" relativi soprattutto all'uso degli 
stent; c'è chi li utilizza con buoni risultati e chi li rifiuta aprioristicamente sostenendo l'inefficienza, peraltro non comprovata, degli stessi. La letteratura nonché le casistiche di alcuni centri con esperienza pluriennale danno torto a coloro che li rifiutano; se si va, poi, ad analizzare il loro modello organizzativo, non è difficile scoprire l'assenza di una collaborazione vera fra loro e il nefrologo che si è sempre limitato al semplice invio dei pazienti.

\section{Lo strumento "strategia"}

Atteso che chirurghi vascolari e angioradiologi interventisti siano ormai strumenti indispensabili nella gestione degli accessi, sta alla competenza e alla capacità del nefrologo fare in modo che le due discipline non siano in contrapposizione o alternative fra di loro; ciascuna va chiaramente messa in campo con indicazioni che, in prima battuta, toccano proprio al nefrologo. L'ideale viene raggiunto quando il nefrologo riesce a far interagire i vari specialisti arrivando con loro alla definizione di un progetto collegiale che identifichi gli attori dei vari step procedurali. La massima espressione del buon funzionamento del team viene raggiunta sia con la partecipazione diretta del nefrologo, anche solo in veste di spettatore, all'allestimento dell'accesso vascolare, che, con quell'atto, dà al paziente e ai colleghi un forte segnale di presenza, sia con l'informazione al personale infermieristico sulle procedure eseguite e sulle necessarie misure da adottare per il corretto utilizzo dell'accesso allestito.

Nella gestione dell'accesso vascolare, in qualunque fase ci si trovi, il segreto per avere maggiori possibilità di successo è quello di giocare il più possibile di anticipo; così, per esempio, mentre si sta allestendo l'accesso utile per quella circostanza, è fondamentale progettare per lo stesso paziente quello che, a grandi linee, sarà il suo prossimo accesso. Il suggerimento, ormai ricorrente nelle varie occasioni scientifiche, è quello di sapere già oggi che cosa potremo fare domani per assicurare l'accesso vascolare ai nostri pazienti e, soprattutto, se saremo in grado di provvedervi da soli. Progettare assume anche il significato di prevedere ovvero di immaginare quale potrebbe essere la prossima difficoltà e la strategia per superarla. Quanto più incerto e oscuro può apparire il futuro di un paziente, relativamente al suo accesso vascolare, tanto maggiore e accurata deve essere la sorveglianza dell'accesso che sta utilizzando; in questo diviene fondamentale il ricorso allo strumento “ infermiere ". Si potrebbe, pertanto, dire che il kit di gestione dell'accesso vascolare non è mai confinato in qualche angolo del centro dialisi ma è sempre pronto ed efficiente come il carrello dell'urgenza che sta subito fuori dalla porta della sala.

\section{C'è posto nel kit per altri strumenti?}

È evidente che la costituzione del team degli accessi possa essere più facilitata dalla presenza di specialisti nella stessa azienda ospedaliera in cui opera il centro dialisi e questo, in genere, può avvenire nei grandi complessi ospedalieri. La mancanza di utili supporti locali non preclude però la costituzione di un team dell'accesso, magari diverso, ma sempre funzionale al raggiungimento degli stessi obiettivi. Il posto degli specialisti mancanti nella struttura in cui opera il nefrologo deve essere utilmente occupato da figure che possono comunque perseguire le finalità del team; ci riferiamo, per esempio, a tutti quei quadri dirigenziali che possono non solo autorizzare ma anche incentivare, quando occorre, il trasferimento del paziente laddove può essere trattato. Non è utopia, bensì semplice applicazione di provvedimenti ispirati all'etica. È bene rimarcare che la responsabilità di individuare e seguire il percorso più utile nella gestione dell'accesso vascolare non può più gravare sul solo nefrologo ma deve gravare sull'intero team degli accessi, qualunque sia la sua composizione.

\section{Per concludere}

Il team dell'accesso è un insieme di professionalità e competenze che condivide un'unica missione: risolvere i problemi dell'accesso vascolare. Come ogni team, riconosce un leader, inteso come colui che cura la regia e che controlla che tutti i componenti perseguano lo stesso fine, seppur con modalità diverse; il team-leader dell'accesso vascolare per la dialisi non può che essere il nefrologo dedicato.

\section{Riassunto}

Gestire l'accesso vascolare oggi è sempre più difficile per il nefrologo, soprattutto se deve farlo da solo. Il team dell'accesso vascolare è uno strumento importante del quale ciascun nefrologo dovrebbe dotarsi; è costituito da varie componenti, a iniziare da quella infermieristica "strutturalmente" già presente in un centro dialisi. Chirurgo vascolare e angioradiologo interventista sono ormai alleati irrinunciabili per il nefrologo che deve affrontare i problemi sempre più complessi dell'accesso vascolare. I componenti del team devono poter interagire fra loro sotto la sapiente regia del nefrologo, la cui cultura sul tema deve essere la più ampia, completa e aggiornata possibile. L'impossibilità di creare il team dell'accesso per mancanza di specialisti nella struttura in cui opera, non deve mai però impedire al nefrologo di individuare altri team esterni a cui fare riferimento per proporre i suoi casi complessi.

Parole chiave: Accesso vascolare, Chirurgo vascolare, Angioradiologo interventista

Dichiarazione di conflitto di interessi: Gli Autori dichiarano di non avere conflitto di interessi.

Indirizzo degli Autori:

Dr. Franco Galli

C.so Garibaldi $20 \mathrm{~m}$

27100 Pavia

frabiagio@gmail.com 